\title{
Colaboraciones técnicas y políticas trasatlánticas: América Latina y la OIT $(1928-1946)^{*}$
}

\author{
Technical collaborations and transatlantic \\ policies: Latin America and the ILO \\ (1928-1946)
}

\author{
Patricio Herrera González**
}

\begin{abstract}
Resumen
Desde Ginebra, la Oficina Internacional del Trabajo se interesó desde muy temprano por vincularse con técnicos, profesionales, intelectuales y dirigentes sindicales del continente americano. Las relaciones entre América Latina y la propia OIT, hoy sigue siendo una temática que presenta muchas opacidades en la historiografía de las relaciones internacionales, laborales e institucionales. Esta investigación explora la relación que establecieron Moisés Poblete Troncoso (chileno) y Vicente Lombardo Toledano (mexicano), que es un ejemplo de muchas otras, y que es hora de describir y explicar para interpretar de mejor forma la institucionalidad laboral de América Latina y el Caribe, ampliando las perspectivas que han centrado su
\end{abstract}

Este trabajo se inscribe en el marco del proyecto FONDECYT Iniciación No11140839, patrocinado por Conicyt y la Universidad de Valparaíso.

** Doctor en Historia, Centro de Estudios Históricos, El Colegio de Michoacán, Zamora, México. Profesor-investigador titular en la Facultad de Ciencias Económicas y Administrativas, Universidad de Valparaíso, Chile. Correo electrónico: patricio.herrera@uv.cl. Recibido: 4 de noviembre de 2016. Aceptado: 18 de julio de 2017. 
atención exclusivamente en las "prácticas obreras".

Palabras clave: Vicente Lombardo Toledano - Moisés Poblete - OIT - América Latina - Legislación Social - Colaboración.

\section{Abstract}

From Geneva, the International Labour Office was interested from early linked to technicians, professionals, intellectuals and union leaders in the Americas. Latin America and the ILO itself, today remains a subject that has many opacities in the historiography of international, labor and institutional relations. This research explores the relationship established Moisés Poblete Troncoso (Chile) and Vicente Lombardo Toledano (Mexico), which is an example of many others, it is time to describe and explain, to interpret to better labor institutions in Latin America and the Caribbean, expanding perspectives have focused exclusively on the "labor practices".

Keywords: Vicente Lombardo Toledano - Moisés Poblete, ILO, Latin America, Social Legislation, Collaboration. 


\section{INTRODUCCIÓN}

Desde Ginebra, la Oficina Internacional del Trabajo se interesó desde muy temprano por vincularse a técnicos, profesionales, intelectuales y dirigentes sindicales del continente americano. En ese marco, la relación entre Vicente Lombardo Toledano y Moisés Poblete no puede extrañarnos, pero a pesar de que las fuentes demuestran lo importante que ambos fueron para América Latina y la propia Organización Internacional del Trabajo, hoy sigue siendo una temática que presenta muchas opacidades en la historiografía de las relaciones laborales e institucionales. Esta contribución explora la relación que establecieron el dirigente obrero y el funcionario ginebrino, ejemplo de muchas otras, que es hora de describir y explicar para interpretar de mejor forma la institucionalidad laboral de América Latina y el Caribe, ampliando las perspectivas que han centrado su atención exclusivamente en las "prácticas obreras".

La investigación sobre los vínculos de funcionarios y técnicos de la Organización Internacional del Trabajo (OIT) con dirigentes del movimiento obrero latinoamericano es un estímulo para iniciar estudios que nos ayuden a situar de mejor manera las relaciones políticas, socioeconómicas y laborales experimentadas por los trabajadores en el segundo tercio del siglo XX, ampliando el enfoque de colaboración entre el Estado, los patrones y los obreros (Van der Linden, M.; Lucassen, J.; Bertrams, K.; Kott, S.; Van Daele, J.; LespinetMoret, I.; Viet, V.) ${ }^{1}$.

La historiografía obrera sigue sin ocuparse del protagonismo que tuvo la OIT en la transferencia de experiencias y conocimientos en materia de legislación laboral, sindicalización y conciliación en América Latina. Esto explica, en parte, el sesgo en la crítica que ha existido por parte de los historiadores del movimiento obrero (Alba, V.; Godio, J.; Rama, C.; Melgar, R.; Alexander, R.) ${ }^{2}$ hacia la temprana

Investigaciones recientes promueven una renovación de los estudios del trabajo y la clase obrera, considerando las relaciones internacionales y la participación de las organizaciones internacionales en la cooperación para instaurar políticas sociales a favor de la protección social y laboral. Los trabajos refieren específicamente a la realidad europea, pero son un punto de partida para repensar la situación de América Latina.

2 Las historiografías obreras, regionales y nacionales, han reproducido a lo largo de las décadas una perspectiva lineal con respecto al carácter revolucionario e intransigente de la clase obrera, sin presentar quiebres en sus principios, posiciones y estrategias. En el caso de los Estados nacionales, estos fueron representados como represores permanentes, incapaces de elaborar una agenda socio-laboral para producir un equilibrio en las relaciones del capital-trabajo. Por último, la clase dueña del capital fue 
legislación social, y los atributos de inoperancia que se le han asignados a órganos públicos, como las oficinas o departamentos del trabajo, y a funcionarios gubernamentales o internacionales, como por ejemplo el caso de Moisés Poblete (Chile), Alejandro Unsain (Argentina), Afonso Bandeira de Mello (Brasil) y José Enrique Sandoval y Saavedra (Cuba). Como resultado de ello, se ha descuidado la investigación sobre los modernos mecanismos de control laboral y la regulación de las relaciones laborales, en un contexto de creciente intervención social de raíces nacionales e internacionales (Herrera, P.; Van Daele, J.; Seekings, J.; Rodríguez, M.; Herrera, F.; Wehrli, Y.; Lespinet-Moret, I.; Viet, V.; Yáñez, J.; Ackerman, M.; González, A. ${ }^{3}$ ).

caracterizada como indolente y desinteresada en el bienestar social de los obreros. Hay que reconocer que si bien estos puntos de vista se encuentran en las acciones acometidas por algunas de las tres partes antes señaladas, no es menos cierto que hubo otro tipo de relaciones, que implicaron una integración y cooperación entre ellos, lo cual en ningún caso significó renunciar a convicciones, pero supuso modificar posiciones o tácticas para lograr los objetivos propuestos.

3 Es importante subrayar que en el presente aún existen vacíos historiográficos en relación a las actividades y políticas que se implementaron entre los países de América Latina y la OIT. Recientemente hay algunos estudios que han realizado un estado
La OIT debió lidiar con los totalitarismos -fascismo, nazismo, comunismo- desarrollados en Europa. Además, sus resoluciones y convenios fueron cuestionados como parte de una política liberal fracasada. Por tanto, la iniciativa de universalizar sus principios de legislación laboral y social presentó un duro revés cuando se inició la conflagración entre las potencias europeas, pues su campo de acción se vio drásticamente disminuido y eventualmente sin interlocutores.

No fue casual, entonces, que la OIT dirigiera sus miradas hacia América, territorial y políticamente neutral por un prolongado tiempo, en comparación con las potencias europeas enfrentadas en un conflicto devastador. Para la OIT significó poder encauzar su misión universalista. En este sentido, la situación laboral de América Latina le proporcionó un impulso a sus principios, pues aquí descubrió problemas que no había percibido en Europa, tales como la exclusión del campesinado de la protección laboral, el estado de pobreza en importantes masas indígenas y la imprevisión en la que vivían cientos de miles de hombres y mujeres a lo largo y ancho del continente

del arte o síntesis de los contactos, donde se demuestra la escasa atención prestada a esta organización internacional, en el marco de las relaciones transnacionales. 
(OIT, Actas II, 1940; OIT, Actas III, 1946).

La OIT, como agencia internacional, fue fundamental para garantizar cohesión y evitar conflictos sociales globales. En ese contexto, la presencia del organismo internacional en el continente es indicativo de una época que exigió fórmulas nuevas para resolver las demandas de la productividad y el bienestar social. Hacia mediados de los años treinta, los países de la región estaban acumulando muchas tensiones y las acciones represivas o revolucionarias, como antaño, parecían no tener cabida. En esto coadyuvó la estrategia de colaboración interclases, promovida por la Internacional Comunista, resultado de la resolución sancionada en el VII Congreso de la Internacional, celebrada en Moscú en agosto de 1935.

La presencia de la OIT permitió abrir nuevos cauces, pero fue necesaria la voluntad de un movimiento de trabajadores dispuesto a negociar y la apertura de agencias obreras o gubernamentales, para emprender estudios técnicos sobre diversas materias laborales, económicas y sociales, tales como el nivel de los salarios, la integración indígena, la situación laboral y social del campesinado, la seguridad social, la inserción de la medicina del trabajo, la educación técnica o la participación política de los trabajadores. En concreto, se trató de instaurar una agenda laboral y social que estaba plenamente vigente en el debate sindical, patronal y estatal, pues había problemas estructurales aún sin solución efectiva.

Los vínculos de la OIT y América Latina se pueden observar, a microescala, en la estrecha relación profesional, gremial y de amistad que se estableció entre el dirigente sindical Vicente Lombardo Toledano (mexicano) y Moisés Poblete (chileno), funcionario de la OIT. Las fuentes de archivo proporcionan antecedentes -inéditos- sobre la circulación de materias técnicas del trabajo y legislación social, como también detallados informes de la situación política y sindical en la región, que permitieron a ambos definir posiciones técnicas y políticas, y colaborar por una agenda laboral y socioeconómica para el continente.

\section{Primeros intercambios}

La participación de Vicente Lombardo Toledano en las diversas esferas de la vida pública de México, América Latina y Europa, aún espera por una investigación rigurosa que integre sus experiencias como intelectual, dirigente obrero y líder político. Entre sus múltiples aportaciones como dirigente obrero, se le ha prestado una menor atención a la estrecha relación que mantuvo con altos funcionarios de la OIT, al menos por dos décadas. Vínculos que, con el correr del 
tiempo, fueron primordiales para establecer un contacto regular entre los funcionarios de la OIT y los dirigentes de la Confederación de Trabajadores de México (CTM), y posteriormente con los líderes de la Confederación de Trabajadores de América Latina (CTAL).

El líder sindical conoció de primera mano las funciones de la OIT. Su profesión de abogado, sus amistades con la clase política revolucionaria, su participación como dirigente en la Confederación Regional Obrera Mexicana (CROM) y sus vínculos con intelectuales de México, América Latina y Europa, le permitieron participar en varias instancias donde pudo tener contacto regular con los funcionarios de la OIT.

La exclusión de México, como resultado de su proceso revolucionario, en el nuevo orden internacional que sancionó el Tratado de Versalles y las Conferencias de Washington, no fueron impedimento para que las organizaciones internacionales de Ginebra, Sociedad de Naciones (SDN) y OIT, y algunas personalidades relevantes de la política mexicana, iniciaran un "acercamiento mutuo y excepcional", dando "pie a una serie de intercambios y tentativas dirigidas a formalizar su colaboración" (Herrera, F., 2011: 336; González, A.). En ese contexto, Vicente Lombardo Toledano fue considerado un " amigo que está destinado a convertirse en uno de nuestros mejores colaboradores con el presidente Calles y la Confederación General de Trabajadores de México" ${ }^{4}$, afirmó un funcionario de la OIT.

Antonio Fabra Ribas, con residencia en Madrid, fue corresponsal de la OIT para América Latina. Nexo recurrente para vincular al dirigente obrero mexicano con otros funcionarios residentes en Ginebra. En innumerables ocasiones solicitó a jefes de Servicios de la OIT, tales como Edgar Milhaud o Robert Boisnier, enviar a Lombardo Toledano documentos mimeografiados de las comisiones de seguros, accidentes de trabajo y enfermedades profesionales; informes provisorios de las conferencias internacionales en francés y español; las listas de delegados definitivos a las conferencias, y los estudios sociales y económicos que se elaboraban en la Oficina Internacional del Trabajo5

4 Archivos de la Oficina Internacional del Trabajo (en adelante AOIT), Antonio Fabra Ribas a Edgard Milhaud, Ginebra, 2 de junio de 1925, Exp. RL 41/3/2: "Relations with the Confederacion de Trabajadores de Mexico". AOIT, Edgard Milhaud a Vicente Lombardo Toledano, Ginebra, 5 de junio de 1925, Exp. I-B12; AOIT, A. Fabra Ribas a Robert Boisnier, Ginebra, 12 de junio de 1925, Exp. RL 41/3/2: "Relations with the Confederacion de Trabajadores de Mexico"; AOIT, Robert Boisnier a Vicente Lombardo Toledano, Ginebra, 19 de junio de 1925, Exp. RL 41/3/2: "Relations with the Confederacion de Trabajadores de Mexico". 
El interés del sindicalista mexicano por los estudios sobre la cuestión del trabajo y las políticas laborales, en una dimensión integral -legislativa, sindical y científica-, lo llevó a representar a la delegación obrera mexicana en la Conferencia Internacional del Trabajo, realizada en Ginebra en el año 1925, cuestión que lo situó como un interlocutor legítimo entre la dirigencia de la clase obrera mexicana y la OIT. En estos certámenes conoció a Francisco Largo Caballero, Ramón González Peña, Amaro del Rosal (UGT España); León Jouhaux (CGT Francia); Eduardo Fimmen (Federación Sindical Internacional), y John Lewis (CIO USA), con los cuales sostuvo relaciones regulares, acompañando muy de cerca su trabajo sindical en México y América Latina.

Su participación en la delegación mexicana durante la celebración de la Conferencia Internacional del Trabajo en Ginebra (1925), permitió a Lombardo Toledano iniciar una relación con varios funcionarios de la OIT. En ese contexto, le manifestó explícitamente a Robert Boisnier que existía en todos sus compañeros "el mejor deseo de estrechar relaciones con esa Oficina”, puntualizando que buscaría la "forma en que la Confederación Regional Obrera Mexicana” cooperara con la OIT $^{6}$.

AOIT, Vicente Lombardo Toledano a Robert Boisiner, Ciudad de México, 15 de agosto de 1925, Exp. RL 41/3/2:
En el año 1926, en el mes de junio, el Consejo de Administración de la Oficina Internacional del Trabajo inscribió la cuestión relativa a la libertad sindical, materia que se discutiría en la Conferencia Internacional del Trabajo del verano de 1927. Fernando Maurette, jefe de la división de Investigaciones de la Oficina, no disponía de información para el caso mexicano, reconociendo que se encontraba imperfectamente documentado al respecto. Maurette, a sugerencia de Antonio Fabra Ribas, escribió a Vicente Lombardo Toledano para que le proporcionara "un estudio completo respecto de la evolución [sindical] en México", pues en la Oficina Internacional importantes funcionarios conocían de su "alta competencia" (Lombardo Toledano, 1926: 16) ${ }^{7}$. Lombardo Toledano correspondió a la solicitud de Fernando Maurette con un estudio muy completo, titulado La libertad sindical en México (1926), manifestando sentir "agradecimiento por la atención de que he sido objeto por parte de esa Oficina y, como siempre, me suscribo a sus órdenes" (Lombardo Toledano, 1926: 20) ${ }^{8}$.

"Relations with the Confederacion de Trabajadores de Mexico".

Fernando Maurette a Vicente Lombardo Toledano, Ginebra, 25 de junio de 1926.

8 Fernando Maurette a Vicente Lombardo Toledano, Ginebra, 25 de junio de 1926. 


\section{COlaboraciones}

TRASATLÁNTICAS

Moisés Poblete Troncoso, abogado chileno y académico internacional, experto en legislación laboral y social, fue un reconocido funcionario de la Oficina Internacional del Trabajo, jefe del Departamento de Problemas Latinoamericanos, que se ocupó de preparar detallados estudios técnicos y sociales, resultado de sus investigaciones y viajes a terreno, que tuvieron por objetivo identificar los principales rasgos del derecho del trabajo y ordenamiento socioeconómico del continente, materias que la OIT necesitaba conocer para desarrollar su misión universalista y que Poblete supo proporcionar con rigurosa sabiduría (Yáñez, 2013: 25-61).

Moisés Poblete y Vicente Lombardo Toledano mantuvieron un estrecho contacto y colaboración entre 1928-1946. La relación entre ambos supuso intercambios profesionales, técnicos, políticos y de amistad, que les permitió compartir diagnósticos sobre problemas laborales, económicos, indígenas, agrarios y sindicales del continente. Se conocieron en la VI Conferencia Internacional Americana celebrada en La Habana, Cuba, entre el 16 de enero y el 20 de febrero de 1928. En el mes de diciembre de ese año, el dirigente sindical mexicano le escribió a Moisés Poblete una atenta misiva, señalando: "me permito recordar a usted el amable ofrecimiento que me hizo en La Habana, de enviarme la edición de las leyes del trabajo de los países latino-americanos [sic], que va precedida con el interesante estudio que hizo usted para ella" La solicitud de Lombardo Toledano fue correspondida por el funcionario de la OIT, enviando el primer volumen de la Legislación social de América Latina (1928), obra coordinada por él y financiada por la Oficina Internacional del Trabajo.

Poblete Troncoso aprovechó la correspondencia mutua para manifestar al dirigente mexicano que estaba al corriente de la iniciativa del Presidente Portes Gil para someter a discusión, en una gran Convención, un proyecto de Código Federal del trabajo, puntualizando al respecto: "he seguido con vivo interés los incidentes de esa Convención, en la que Ud. [refiriéndose a Vicente Lombardo] tomará una participación tan activa y útil por su preparación en materia social” ${ }^{10}$. Esta situación, acontecida en México, impidió que se conocieran las leyes laborales del país en el segundo volumen de la Legislación Social de América Latina (1929), pues Poblete consideró inoportuno

AOIT, Vicente Lombardo Toledano a Moisés Poblete, Ciudad de México, 21 de diciembre de 1928, Exp. I-B12. 10 Moisés Poblete a Vicente Lombardo Toledano, Ginebra, 31 de enero de 1929, Exp. IB12 
imprimir una legislación que estaba en proceso de cambio.

Con el correr de los meses, la discusión sobre el Código Federal del Trabajo en México se fue decantando. Vicente Lombardo estuvo pendiente de la aprobación del Código y en cada carta enviada a Moisés Poblete le comentaba que tan "pronto esto ocurra, tendré el gusto de enviarle algunos ejemplares" ${ }^{11}$. Poblete, por su parte, abrigaba esperanzas para que eso ocurriera, pues tenía un estudio que la Oficina publicaría y distribuiría. Él concebía que la promulgación del Código Federal del trabajo facilitaría la "adhesión de México a la Organización Internacional del Trabajo", considerando que el país exhibía un "avanzado desarrollo social”, por lo que resultaría beneficioso para ambas partes "su próximo ingreso como miembro de la misma" 12 .

En el mes de octubre de 1930, y como parte de la política de acercamiento hacia América del nuevo director de la OIT, Harold

11 AOIT, Vicente Lombardo Toledano a Moisés Poblete, Ciudad de México, 29 de agosto de 1929, Exp. RL 41/3/2: "Relations with the Confederacion de Trabajadores de Mexico".

12 Moisés Poblete a Vicente Lombardo Toledano, Ginebra, 7 de abril de 1930, Exp. RL 41/3/2: "Relations with the Confederacion de Trabajadores de Mexico". Finalmente, en el mes de agosto de 1931 se promulgó el Código Federal del Trabajo en México.
Butler, Moisés Poblete fue invitado, por recomendación de Lombardo Toledano, a la Universidad Nacional Autonoma de México a dictar una serie de conferencias sobre "La evolución social de la América Latina"13. La amistad y colaboración entre el funcionario internacional y el dirigente sindical mexicano se acrecentó a partir de ese momento.

Durante su estancia en México, Poblete Troncoso se convenció que el país sería un buen promotor de los proyectos e iniciativas de la OIT. La proximidad con los Estados Unidos y sus vínculos con los países de Centroamérica y el Cono Sur, garantizaban una corriente de cooperación y diálogos sobre los asuntos del trabajo. Basta recordar que México se incorporó a la OIT en 1931, y Estados Unidos lo concretó en 1934. De ahí que México fue un eje de circulación y caja de resonancia en el continente, para dar a conocer los informes sociales, estudios técnicos y resoluciones de la OIT en su primera década. Al respecto, Poblete envió artículos a Vicente Lombardo para que los

13 Fondo Histórico Lombardo Toledano (en adelante FHLT), Id. 9874, legajo 174. 
"haga publicar en El Universal"14 y la revista Futuro ${ }^{15}$.

En el primer trimestre de 1931, el sindicalista mexicano visitó algunos países del Cono Sur, participando como invitado en congresos de maestros y estudiantes universitarios. En Buenos Aires se reunió con Nicolás Repetto, Francisco Pérez Leirós y Enrique Dickmann, dirigentes del Partido Socialista. En Montevideo se entrevistó con Emilio Frugoni, líder del Partido Socialista de Uruguay. En Chile, a sugerencia de Moisés Poblete ${ }^{16}$, fue recibido por Francisco Walker Linares, profesor de Economía Social de la Universidad de Chile,

14 Moisés Poblete a Vicente Lombardo Toledano, Ginebra, 7 de abril de 1930, Exp. RL 41/3/2: "Relations with the Confederacion de Trabajadores de México".

15 Los artículos decían relación con la legislación social, los problemas del trabajo y la situación política del nazismo y sus consecuencias para el orden internacional. Fueron publicados en el año 1934: "Una gran figura que desaparece del escenario ruso: Anatole Lounatscharsky", Futuro, primera quincena de marzo de 1934, p. 26; "La defensa de la raza", Futuro, primera quincena de abril de 1934, p. 11; "El ocaso de una civilización", Futuro, segunda quincena de abril de 1934, p. 12; "El porvenir de los trabajadores alemanes. La carta del trabajo nacista", Futuro, mayo de 1934, p. 17

16 Carta de Moisés Poblete a Vicente Lombardo Toledano, Ginebra, 21 de febrero de 1931, FHLT, Id. 9983, legajo 179 . sosteniendo reuniones con algunos líderes de las corrientes del socialismo chileno. Estos encuentros serían decisivos en el liderazgo sindical continental de Vicente Lombardo Toledano, luego de 1938, dado que su red de apoyo político con varios de estos dirigentes, de corriente socialista, cooperó en allanar el camino entre las organizaciones obreras.

Al finalizar 1932, la CROM se dividió por razones de principios y línea estratégica. La mayoría de sus organizaciones aprobó un nuevo programa de acción, redactado por Vicente Lombardo Toledano, dando vida a la Confederación General de Obreros y Campesinos de México (CGOCM), en el mes de octubre de 1933, que entre sus orientaciones principales tomó la resolución de cooperar para la "unidad de los trabajadores de la América Latina" (Lombardo Toledano, 1995: 232). Poblete estaba al corriente del quiebre de la CROM, por eso le solicitó a Lombardo Toledano los mayores antecedentes de esta situación, "porque sigo con gran interes [sic] y apasionamiento el movimiento obrero de su país, que me parece el mejor coordinado y el más fuerte de todos los países de América" 17.

17 AoIT, Moisés Poblete a Vicente Lombardo Toledano, Ginebra, 12 de noviembre de 1932, Exp. RL 41/3/2: "Relations with the Confederacion de Trabajadores de Mexico". 
A esas alturas, los conocimientos técnicos -sobre temas laborales, la situación socioeconómica y política de América Latina y el Caribe- de Moisés Poblete y Vicente Lombardo Toledano habían consolidado una relación de colaboración, a tal grado que fue muy frecuente que intercambiaran bibliografías e informes manuscritos de primer orden, que luego se materializaron en libros y estudios. Por ejemplo, Moisés Poblete publicó en México El movimiento obrero latinoamericano (1946), primer esfuerzo de síntesis histórica sobre la formación y evolución del movimiento obrero de la región, trabajo pionero en su tipo. El libro desarrolló una interpretación con minuciosos detalles. Para la historiadora norteamericana S. Fanny Simon (1946: 550), Poblete ofrece una investigación sugerente. A su juicio, la base de la información del libro fue proporcionada por Lombardo Toledano, cuestión que coincide con los estudios sobre México y América Latina que elaboró el dirigente sindical y los antecedentes que reunió en sus viajes y reuniones con agrupaciones obreras, cuando lideró las diversas organizaciones obreras de México (CROM, CGOCM, CTM) y América Latina (CTAL).

Con su libro, Poblete contribuyó a la formación de una comunidad obrera internacional e instaló un modelo de institucionalidad laboral, promoviendo la justicia social a través de una vía reformista. Su idea fue abrir espacios para una efectiva participación de todas las fuerzas sociopolíticas del continente, con el fin de consolidar la democracia electoral y representativa.

\section{UNA AGENDA SOCIOLABORAL COMÚN}

Vicente Lombardo Toledano fue consciente de la importancia del movimiento obrero mexicano para iniciar la unidad de los obreros en el continente. Su intención fue preparar intelectualmente a los dirigentes obreros con el fin de renovar sus cuadros políticos y "empoderar" a las masas. Para formar a los obreros proyectó una universidad obrera. Por esta razón, solicitó al jefe de Servicio de Relaciones Obreras de la OIT, Adolf Staal ${ }^{18}$, le informara

18 Adolf Staal nació en Ámsterdam (Países Bajos), en 1886. Estudió lenguas en sus ratos de ocio, convirtiéndose en un importante traductor de idiomas (inglés, francés, alemán y español). Fue uno de los primeros miembros del sindicato de trabajadores del comercio en los Países Bajos, cuyo secretario general fue E. Fimmen. Cuando la Federación Sindical Internacional (FSI) se reconstituyó, luego de 1919, E. Fimmen se transformó en el secretario general, nombrando a Adolf Staal como traductor y jefe de Personal de la FSI. Adolf Staal asistió a todas las Conferencias Internacionales del Trabajo después de 1921, desempeñándose como secretario de la delegación de los obreros. En el mes de septiembre del año 
lo "más ampliamente posible sobre lo que se ha hecho en los países europeos en materia de educación obrera". Además, lo exhortaba para que le envíe los "planes de estudios de las principales instituciones educativas que existen en las naciones más avanzadas de Europa para la educación sindical, política y cultural de las masas"19. Adolf Staal respondió prontamente al dirigente obrero mexicano, adjuntando un proyecto de estudio sobre esta cuestión, afirmando: "estoy seguro que ha de encontrar en esos docu-

1929, Albert Thomas le ofreció un contrato en la OIT y fue designado jefe de Servicio de Relaciones Obreras Internacionales, con una remuneración de 19.000 francos suizos anuales. Durante su gestión, en 1946 se jubiló, tuvo contacto y estrechos vínculos con dirigentes y movimientos obreros de Europa, África, Asia y América Latina. Con respecto a la CTAL, estuvo informado desde 1936 de la formación de una confederación obrera en América Latina. Asistió como representante de la OIT al Congreso Obrero Latinoamericano, México 1938; al Primer Congreso de la CTAL, México 1941; y al Segundo Congreso de la CTAL, Colombia 1944. También estuvo presente en la primera y segunda Conferencia del Trabajo de los Estados de América (Santiago de Chile, 1936; y La Habana, Cuba, 1939), a cargo del servicio de relaciones con las delegaciones obreras. AOIT, Exp. P 2310: “A. Staal du service du personnel".

19 AOIT, Vicente Lombardo Toledano a Adolf Staal, Ciudad de México, 28 de febrero de 1933, Exp. I-B12. mentos muchos datos útiles sobre la materia" 20 .

Vicente Lombardo Toledano inauguró la Universidad Obrera de México el 8 de febrero de 1936, concebida como un referente intelectual para la clase proletaria de México y el continente. Desde sus aulas, concibió un "nuevo amanecer" para los trabajadores, tal como quedó de manifiesto en el discurso inaugural: "tengo la convicción de que [...] la Universidad Obrera de México no es más que un paso que se anticipa unos cuantos días a la unidad definitiva del movimiento proletario" (CTM, 1941: 85).

Entre el 2 y 14 de enero de 1936, en Santiago de Chile, durante la primera Conferencia Americana del Trabajo (Herrera, 2013a: 199-242), organizada por la OIT y el gobierno de Arturo Alessandri Palma, le correspondió a Moisés Poblete oficiar como coordinador del encuentro, por su conocimiento administrativo de la organización internacional y de su país natal.

En tanto, un directo y provocador mensaje de Vicente Lombardo Toledano-quien no pudo asistir a la conferencia en Chile- a los delegados, particularmente a los obreros, recordaba la situación económica y social de los trabajadores y de los pueblos latinoamericanos, realizando un llamado a la clase obrera

20 Adolf Staal a Vicente Lombardo Toledano, Ginebra, 29 de marzo de 1933, Exp. I-B12. 
para su unificación y contribución al progreso de las naciones "semiindependientes" (El Universal, México, 1 de enero 1936) ${ }^{21}$. Este mensaje contribuyó a promover la unidad de los trabajadores del continente. Recordemos que al margen de la conferencia, varios obreros que oficiaron como delegados y otros que acompañaron a sus representantes, firmaron un compromiso para promover la unidad de los trabajadores en una organización sindical de alcance continental ${ }^{22}$ (Herrera, 2013b: 87-119), que dos años más tarde se materializó con la formación de la CTAL.

La participación de Adolf Staal, jefe de Servicio de Relaciones Obreras de la OIT, como integrante de la Comisión de Servicios de Relaciones con las Delegaciones durante la conferencia, manifestó el interés que tuvo desde un inicio la Organización Internacional por establecer un trabajo conjunto con los obreros de América Latina.

21 Lombardo celebra la primera Conferencia Americana del Trabajo, organizada por la OIT, en Santiago de Chile. En su mensaje promueve enfáticamente la idea de formar una gran confederación obrera para el continente.

22 Francisco Pérez Leiros, Pedro Chiaranti y Antonio Sánchez, por Argentina; Elías Lafferte, Luis Solís, Juan Díaz Martínez e Isidoro Godoy por Chile; Felipe Ortiz por Bolivia; Rafael Burgos por Colombia; Mario Masi por Paraguay; Arturo Freire y José Lazarraga por Uruguay; y Rosendo Naula por Ecuador.
De hecho, Staal reconoció, en su reporte anual como funcionario de la OIT, que la reunión en Santiago permitió "contactos con las organizaciones obreras, valiosas para ambas partes" 23 . Incluso, en los meses siguientes, el funcionario internacional mantuvo una correspondencia con Vicente Lombardo Toledano y Rodolfo Piña Soria, donde les solicitaba información sobre los preparativos para "un Congreso Obrero Continental [que] vienen realizando desde el Comité Ejecutivo Nacional de la Confederación de Trabajadores Mexicanos (CTM) hace meses", y que para el jefe de Servicio de Relaciones Obreras de la OIT, supuso “el más alto interés en estar cabalmente informado a tiempo de todos los detalles relativos a tan importante proyecto", de ahí su insistencia para que los dirigentes obreros mexicanos le hicieran llegar toda la "documentación eventual y respectiva, así como cuantas informaciones complementarias o aclaraciones personales" ${ }^{24}$ sea oportuno remitir.

23 AOIT, Adolf Staal, Reporte anual de funciones, Ginebra, 24 de septiembre de 1936, Exp. P 2310: “A. Staal du service du personnel".

24 AOIT, Adolfo Staal a Rodolfo Piña Soria, Ginebra, 26 de noviembre de 1936, Exp. RL 41/3/2: "Relations with the Confederacion de Trabajadores de Mexico". La respuesta de Piña Soria a Staal se realizó unos meses después señalando que respecto "al Congreso Latinoamericano, estamos haciendo una activa labor de 
El conocimiento de la reunión obrera continental generó tanta expectativa en la OIT, que fue el mismo Vicente Lombardo quien le escribió a Harold Butler, director de la OIT, para reafirmar que la CTM estaba luchando "por todos los medios a su alcance, a efecto de que los países de la América Latina sigan vinculados a Ginebra, porque ello significa el entendimiento internacional de todo los pueblos" 25 .

Con el tiempo, la OIT estableció una insospechada relación con los dirigentes de los trabajadores de la región, a través de la Confederación de Trabajadores de América Latina, que coincidentemente fue liderada por el mexicano Vicente Lombardo Toledano.

La OIT reconoció la trayectoria del dirigente mexicano en el mes de junio de 1937, en el marco de la $23^{a}$ reunión de la Conferencia Internacional del Trabajo, celebrada en Ginebra, cuando lo nombró miembro adjunto del Consejo de Administración de la Oficina Internacional del

propaganda y sobre este asunto le enviaré los informes necesarios con la oportunidad debida", en AOIT, Rodolfo Piña Soria a Adolf Staal, México, 11 de febrero de 1937, Exp. RL 41/3/2: "Relations with the Confederacion de Trabajadores de Mexico".

25 AOIT, Vicente Lombardo Toledano a Harold Butler, México, 26 de marzo de 1937, Exp. RL 41/3/2: "Relations with the Confederacion de Trabajadores de Mexico".
Trabajo ${ }^{26}$, lo cual fue ratificado con su nombramiento como miembro permanente en dicho Consejo, el 5 de mayo de 1944, durante la $25^{\mathrm{a}}$ Conferencia Internacional del Trabajo (El Popular, México, 6 de mayo 1944). Todo un hito para la historia del movimiento sindical latinoamericano, si consideramos que Vicente Lombardo Toledano fue el primer dirigente obrero de la región en integrar tan importante Consejo, pero que sin desconocer sus méritos propios, no podemos dejar de reflexionar el peso que pudo tener en esas decisiones, su colaboración y amistad con Moisés Poblete, funcionario de la OIT, que por medio de su destacada gestión, como encargado para los asuntos de América Latina, aproximó el valor supremo de la justicia social al continente.

\section{Conclusiones}

Las fuentes disponibles en los archivos dan cuenta de las conexiones internacionales que tuvo la dirigencia obrera del continente con altos funcionarios de la OIT. La presencia regular de delegaciones obreras, transatlánticas y transpacíficas durante la celebración de numerosos congresos internacio-

26 AOIT, Adolf Staal a Vicente Lombardo Toledano, Ginebra, 9 de julio de 1937, Exp. RL 41/3/2: "Relations with the Confederacion de Trabajadores de Mexico". 
nales -Conferencias Internacionales Americanas, Conferencias Internacionales del Trabajo, Conferencias Americanas del Trabajo, Congresos Obreros-, nos invita a pensar la historia latinoamericana en otros registros que, complementados con las aportaciones de las historias nacionales, nos pueden proporcionar reconstrucciones históricas más próximas a la realidad política, social y cultural. A manera de ejemplos, los epistolarios, estudios e informes de Vicente Lombardo Toledano, disponibles en el Fondo Histórico Lombardo Toledano, y los expedientes de los funcionarios de la Oficina Internacional del Trabajo de Moisés Poblete, Adolf Staal o Antonio Fabra Ribas, alojados en el Archivo de la Organización Internacional del Trabajo, reafirman la necesidad de levantar estudios que den cuenta, en forma precisa, de estos vínculos. Todos formaron parte de una red de pensamiento y acción, que si bien no compartieron ideologías y dogmas partidistas, al menos mantuvieron un intercambio, que les permitió colaborar en proyectos que, por coyunturas, parecían aproximarlos en sus posiciones, dejando de lado sus diferencias.

En un principio, la penetración de la OIT en el continente americano fue una acción un tanto espontánea. Los escasos informes técnicos y estudios laborales sobre América Latina antes de 1928, la presencia reducida de funcionarios latinoamericanos en la burocracia de la Oficina Internacional del Trabajo y las mínimas visitas de sus directores a los países de la región -Albert Thomas, su primer director, recién viajó entre 19241925, y posteriormente, en 1934, visitó la región el director Harold Butler-no permitieron un contacto fluido. De hecho, fueron algunos dirigentes sindicales, como el caso de Lombardo Toledano, o funcionarios intermedios de la Oficina Internacional del Trabajo, como Moisés Poblete, quienes cooperaron como mediadores con la clase política gobernante o con los legisladores.

Fue la Conferencia Americana del Trabajo, de 1936, la que inauguró una relación más colaborativa, que en retrospectiva le permitió a la OIT planificar su misión universalista con nuevos derroteros, orientando sus principios de acción, que luego se utilizaron para promover los derechos sociales y laborales en otras regiones del espacio extra europeo, tales fueron los casos de Asia y África en la década de 1960.

En síntesis, el derecho social formó parte de las exigencias laborales de las organizaciones obreras, reivindicación que se acentuó con la formación de la CTAL (Herrera, 2013c). Los debates e informes técnicos, discutidos en cada Conferencia Americana, posteriormente ejecutados en políticas sociales por los gobiernos en acuerdo -aunque a veces bajo presión- con la clase 
obrera organizada, allanaron el camino de reconocimiento del trabajador como sujeto de derecho político, económico y cultural, lo cual modificó sustancialmente la relación capital-trabajo, donde el bienestar de los asalariados, con matices, se intensificó en la agenda de las políticas sociales y legislativas nacionales y continentales.

\section{Anexos}

a. Vicente Lombardo Toledano (1894-1968), mexicano, abogado de profesión. Considerado como uno de los intelectuales más preclaros del país. Su dilatada trayectoria se puede ubicar en tres esferas: sindical, político e intelectual.

En el plano sindical mexicano, latinoamericano y mundial, fue unos de los líderes destacados de la Confederación Regional Obrera Mexicana (CROM) y secretario general de las siguientes confederaciones obreras mexicanas: Confederación General Obrera y Campesina de México (CGOCM) y Confederación de Trabajadores de México (CTM).

También colaboró activamente, junto a otros dirigentes obreros del continente, en la organización del Congreso Obrero Latinoamericano realizado en la ciudad de México, en el mes de septiembre de 1938, en cuyas sesiones los delegados obreros determinaron constituir la Confederación de Trabajadores de América Latina, eligiendo por amplia mayoría a Lombardo Toledano como su secretario general. En 1945 participó en París en el Congreso Obrero Mundial, donde fue elegido, en forma unánime, como primer vicepresidente de la recién creada Federación Sindical Mundial (FSM). Sus esfuerzos siempre estuvieron encaminados a establecer un internacionalismo obrero, lo cual le valió muchas críticas por parte de sus detractores, que lo asociaban a las políticas dictadas por la URSS. Inclusive se le señaló como un agente del comunismo internacional, a pesar de que no militó en el Partido Comunista Mexicano.

Como político, participó en diferentes cargos públicos. Fue gobernador del Estado de Puebla, su tierra natal. También fue electo parlamentario en varias ocasiones y candidato a la Presidencia de la República en 1952, apoyado por el Partido Popular, fundado por el propio Lombardo Toledano. Dado su protagonismo como dirigente sindical y político, estuvo involucrado en numerosos conflictos gremiales y partidistas que lo enemistaron con numerosas organizaciones, tales como el Partido Comunista Mexicano, la American Federation of Labor (AFL) o el gobierno de Estados Unidos, al menos desde el mandato de H. Truman, en el contexto de la Guerra Fría. 
En el plano intelectual, escribió casi un centenar de libros, estudios, ensayos y folletos sobre diversas materias, entre las que destacan: educación, filosofía, derecho, condiciones laborales, derecho laboral, sindicalismo, marxismo, imperialismo, identidad, indigenismo, sufragio femenino, historia de la revolución mexicana, latinoamericana y universal, religión, etc. $\mathrm{Si}$ bien existen importantes estudios sobre la trayectoria de Vicente Lombardo Toledano, aún se espera una investigación acabada de su producción sindical, política e intelectual. Muchos de los estudios no pueden sustraerse a las visiones apologéticas o fustigadoras de su participación como dirigente sindical y líder político ${ }^{27}$.

b. Moisés Poblete Troncoso (18931972) nació en Chillán (Chile). Se recibió de licenciado en Derecho en la Universidad de Chile, donde tuvo una brillante trayectoria académica. Entre sus funciones públicas que desempeñó en Chile destacan: director general del Trabajo; subsecretario del Ministerio del Trabajo. El presidente Arturo Alessandri Palma (1920-1925) le confió la misión de redactar el Proyecto de Código del Trabajo y de la Previsión Social, de conformidad con las aspiraciones

27 FHLT. renovadoras del movimiento popular de 1920. Realizó la primera compilación de leyes laborales y sociales, las cuales fueron entregadas a Albert Thomas, director de la OIT, durante su visita a Chile en 1925. Aquel proyecto constituyó el origen de la mayor parte de la legislación social chilena.

Entre 1927 y 1940 tuvo una destacada posición en el campo social internacional. Albert Thomas, en 1927, lo contrató como experto en legislación social iberoamericana, con un ingreso anual de 15.600 francos suizos. Años más tarde, fue nombrado como funcionario permanente y fue designado como jefe del Grupo Latinoamericano de la Oficina Internacional del Trabajo, en Ginebra. La OIT y la Sociedad de las Naciones le confiaron misiones, entre otras, delegadas a la conferencia de Organización Científica del Trabajo, en Roma, 1929; a la Internacional de Inmigración de La Habana y a la de estructuración de los servicios del Trabajo de Cuba; también se le encomendó el estudio de las condiciones de vida y trabajo de los indígenas en el Perú. Fue el organizador, con notable éxito, de la Primera Conferencia Regional de los Estados Americanos miembros de la OIT, reunida en Santiago en 1936, como también corresponsal de la OIT en Chile. Asimismo, participó como profesor e inves- 
tigador en varias instituciones de educación superior, entre las cuales destacan: la Universidad de Chile; la Société Economie Politique de París; la American Academy of Political and Social Science; el Instituto Internacional de Sociología de Oslo; la Sociedad Internacional de Derecho Social; y fue miembro correspondiente de los institutos de Derecho del Trabajo de Santa Fe, de Córdoba, de la Habana, y del Instituto Mexicano de Derecho Social. Además, publicó un medio centenar de libros y numerosos estudios de legislación social en prestigiosas revistas de Europa y América (Feliú Cruz, 1970; Walker Linares, 1961) ${ }^{28}$.

\section{BIBLIOGRAFÍA}

Ackerman, M. (2000). La Oficina de la OIT para Argentina, Paraguay y Uruguay, 1969-1999. Buenos Aires.

Alba, V. (1964). Historia del Movimiento Obrero en América Latina. México: Libreros Mexicanos Unidos.

Alexander, R. (2009). International Labor Organizations and Organized Labor in Latin America and the Caribbean; A History. Santa Barbara: Praeger/ ABC-CLIO.

Archivo Organización Internacional del Trabajo (AOIT). Ginebra, Suiza.

Bertrams, K. y Kott, S. (2008). Actions sociales transnationales. Genèses, núm. 71, junio, pp. 2-3.

CTM (1941). 1936-1941, tomo I. México: Confederación de Trabajadores de México

28 Véase AOIT, Exp. P 2037: "Moisés Poblete Troncoso".
El Universal, México (1936).

El Popular, México (1936).

Feliú Gruz, G. (1970). Moisés Poblete Troncoso. La bibliografía de las ciencias sociales. Santiago: Biblioteca Nacional.

Fondo Histórico Lombardo Toledano, Ciudad de México.

González Martínez, A. (1986). México y la Organización Internacional del Trabajo. México y la paz. México: Instituto Matías Romero de Estudios Diplomáticos-SRE.

Herrera F. y Wehrli, Y. (2011). Le Bureau international du travail et l'Amérique latine durant l'entre-deux-guerres. Lespinet-Moret, I. y Viet, V. (coords.), L'Organisation internationale $d u$ travail. Rennes: Presses Universitaires de Rennes.

Herrera, F. (2011). México y la Organización Internacional del Trabajo: los orígenes de una relación, 1919-1931. Foro Internacional, núm. 2.

Herrera, F. y Herrera, P. (coords.) (2013). América Latina y la Organización Internacional del Trabajo: redes, cooperación técnica e institucionalidad social (1919-1950). Morelia, México: Universidad Michoacana de San Nicolás de Hidalgo.

Herrera, P. (a) (2013). La primera conferencia regional del trabajo en América; su influencia en el movimiento obrero, 1936. En Herrera, F. y Herrera, P. (coords.), América Latina y la Organización Internacional del Trabajo: redes, cooperación técnica e institucionalidad social, 1919-1950. Morelia. México: Universidad Michoacana de San Nicolás de Hidalgo, pp. 199-242.

Herrera, P. (b) (2013). El pacto por la unidad obrera continental: sus antecedentes en Chile y México, 1936. Estudios de Historia Moderna $y$ Contemporánea de México, núm. 46, pp. 87-119. 
Herrera, P. (c) (2013). "En favor de una Patria de los trabajadores”. La Confederación de Trabajadores de América Latina y su lucha por la emancipación del continente, 1938-1953. Tesis para el grado de Doctor en Historia. Centro de Estudios Históricos, El Colegio de Michoacán, Zamora, México.

Godio, J. (1985). Historia del movimiento obrero latinoamericano, tomo III. San José: Editorial Nueva Sociedad.

Kott, S. (2011). Dynamiques de l'internationalisation: l'Allemagne et l'Organisation internationale du travail (1919-1940). Critique Internationale, núm. 52, julio-septiembre, pp. 69-84.

Kott, S. (2011). Les organisations internationales, terrains d'étude de la globalisation. Jalons pour une approche socio-historique. Critique Internationale, núm. 52, julio-septiembre, pp. 9-16.

Kott, S. (2008). Une 'communauté épistémique' du social? Experts de l'OIT et internationalisation des politiques sociales dans l'entre-deux-guerres. Genèses, núm. 71, junio, pp. 26-46.

Lespinet-Moret, I. y Viet, V. (coords.) (2011). L'Organisation internationale $d u$ travail. Rennes: Presses Universitaires de Rennes.

Lombardo Toledano, V. (1995). Obra Histórico-Cronológica, T. II, Vol. 4. México: CEFPSVLT.

Lombardo Toledano, V. (1928). Bibliografía del Trabajo y de la previsión social en México. México.

Lombardo Toledano, V. (1926). La libertad sindical en México. México: Universidad Obrera VLT.

Lucassen, J. (ed.) (2006). Global Labour History: A State of the Art. Bern: Peter Lang.

Melgar, R. (1988). El movimiento obrero latinoamericano. Historia de una clase subalterna. Madrid: Alianza Editorial.
OIT (1946). Tercera Conferencia del Trabajo de los Estados de América Miembros de la Organización Internacional del Trabajo. Actas de las Sesiones. Montreal: Oficina Internacional del Trabajo.

OIT (1941). Segunda Conferencia del Trabajo de los Estados de América Miembros de la Organización Internacional del Trabajo. Actas de las sesiones. Montreal: Oficina Internacional del Trabajo.

OIT (1929). Legislación social de América Latina, vol. 1. Ginebra: Oficina Internacional del Trabajo.

OIT (1928). Legislación social de América Latina, vol. 1. Ginebra: Oficina Internacional del Trabajo.

Poblete, M. (1946). El movimiento obrero latinoamericano. México: Fondo de Cultura Económica

Revista Futuro, México (1934).

Rodríguez García, M. (2010). Conclusion: The ILO's Impact on the World. En Van Daele, J. et al. (eds.), ILO Histories: Essays on the International Labour Organization and Its Impact on the World During the Twentieth Century. Bern: Peter Lang.

Seekings, J. (2010). The ILO and Welfare Reform in South Africa, Latin America, and the Caribbean, 1919-1950. En Van Daele, J. et al. (eds.), ILO Histories: Essays on the International Labour Organization and Its Impact on the World During the Twentieth Century. Bern: Peter Lang.

Rama, C. (1976). Historia del movimiento obrero y social latinoamericano contemporáneo. Barcelona: Editorial Laia.

Simon, F. (1946). Reseña del libro El movimiento obrero latinoamericano de Moisés Poblete T., publicada en The Hispanic American Historical Review, núm. 26: 4, p. 550.

Van Daele, J. (2010). Writing ILO histories: A State of the Art. En Van Daele, J. et al. (eds.), ILO Histories: Essays 
on the International Labour Organization and Its Impact on the World During the Twentieth Century. Bern: Peter Lang.

Van Daele, J. et al. (eds.) (2010). ILO Histories: Essays on the International Labour Organization and Its Impact on the World During the Twentieth Century. Bern: Peter Lang.

Van Der Linden, M. (2006). Historia transnacional del trabajo. Valencia: Centro Francisco Tomás y Valiente UNED Alzira-Valencia.

Walker Linares, F. (1961). Discurso de recepción del profesor de derecho del trabajo señor Francisco Walker Linares. Anales de la Facultad de Ciencias Jurídicas y Sociales, núm. 3 , Universidad de Chile.

Yáñez, J. (2013). La OIT y la red sudamericana de corresponsales. El caso de Moisés Poblete, 1922-1946. En Herrera, F. y Herrera, P. (coords.), América Latina y la Organización Internacional del Trabajo: redes, cooperación técnica e institucionalidad social, 1919-1950. Morelia, México: Universidad Michoacana de San Nicolás de Hidalgo, pp. 25-61.

Yáñez, J. (2008). La intervención social en Chile. Santiago: RIL Editores.

Yáñez, J. (2000). Chile y la Organización Internacional del Trabajo (1919-1925). Hacia una legislación social universal. Revista de estudios histórico-jurídicos, núm. 22. 